\title{
Toxic Anterior Segment Syndrome (TASS)
}

\author{
Sidi Dahi, Mehdi Khamaily, Joumany Brahim Salem, Wafae Akioud, Houda Brarou, \\ Taoufik Abdellaoui, Yassine Mouzari, Karim Reda, and Abdelbarre Oubaaz
}

\section{ABSTRACT}

Cataract surgery is ranked among the most frequent operations, affecting a population over 65 years of age.Its prognosis is often favorable, however, there are rare complications including inflammation of the anterior segment known as Anterior Segment Toxicity Syndrome . the majority of our patients were men and diabetes appear in almost half and all our patients present various symptoms the first 24 hours dominated by the attack of the anterior segment our therapeutic attitude consists in the use of corticosteroids and hypotonizers with a good evolution in the majority of patients.

Keywords: phacoemulsification, inflammation, Corticosteroid.

\author{
Submitted : November 14, 2020 \\ Published : April 20, 2021 \\ ISSN: $2593-8339$ \\ DOI: $10.24018 /$ ejmed.2021.3.2.571 \\ Sidi Dahi* \\ (e-mail: dahisidi2014@gmail.com) \\ Mehdi Khamaily \\ Joumany Brahim Salem \\ Wafae Akioud \\ Houda Brarou \\ Taoufik Abdellaoui \\ Yassine Mouzari \\ Karim Reda \\ Abdelbarre Oubaaz \\ All authors are with \\ Department of Ophthalmology, \\ Mohammed V Military Teaching \\ Hospital, Rabat, Morocco. \\ Faculty of Medicine and Pharmacy, \\ Mohammed V University, Rabat, \\ Morocco. \\ *Corresponding Author
}

\section{INTRODUCTION}

Cataract surgery is the most frequent operations, affecting a population over 65 years of age. Its prognosis is often favorable, however, there are rare complications including inflammation of the anterior segment known as Anterior Segment Toxicity Syndrom.

\section{MATERIEL AND METHODS}

This is a retrospective study on 10 cases in the ophthalmology service of the Military Instruction Hospital in Rabat between March 2018 - March 2020.

We included in our study, any cataract surgery by phacoemulsification with inflammation of the anterior segment postoperatively.

Our exclusion criteria were Patients followed for uveitis and cases of complicated cataract surgery.

\section{RESULTS}

The patients consisted of 7 men and 3 women of whom 2 had high blood pressure, 4 had type 2 diabetes mellitus, 4 had no significant medical problems, and the phacoemulsification times in all cases were less than 10 minutes.

The overall surgical time for each case did not exceed 30 minutes and the 10 cases developed at different times and did not occur on the same day and the intraocular lenses were hydrophilic acrylic all inserted into the posterior chamber in addition to the intracameral injections of Cefuroxime in all patients Adrenaline in 4 and Pilocarpine in 3, all patients received BSS ${ }^{\circ}$ (Balanced Select solution), and all have corneal stromal edema, visual blurring and inflammatory cells in the anterior chamber the day after surgery (Fig. 1).

Their visual acuity was limited to hand movements or perception of light, moderate eye pain in 3 cases, retrodeskemetic precipitates in 6 and hypopion in 4 , a cyclitic membrane in 04 and ocular hypertonia in 03 cases while the fundus was normal in 2 and inaccessible in 8 patients.

However, B-scan ultrasound showed little inflammation in the vitreous and the inflammation was mostly confined to the anterior segment with no intravitreal organization. Compared to severe inflammation of the anterior chamber, conjunctival inflammation is less severe. This includes conjunctival or ciliary injection, discharge, and conjunctival edema. none of our patients received a puncture of the anterior chamber but steroids at the rate of one drop per hour for $48 \mathrm{~h}$ then progressive reduction in all patients, 
Subconjunctival injection in 4 patients and oral route $1 \mathrm{mg} /$ $\mathrm{kg}$ in 3 patients while hypotonizers: dorsolamide and beta blocker in 3 patients, the evolution after 3 months postoperatively shows in 7 patients a clear cornea while the corneal edema persists in 2, a decompensated patient candidate for keratoplasty and acuity visual more or equal to $5 / 10$ in 7 and less than $5 / 10$ in 3 patients .

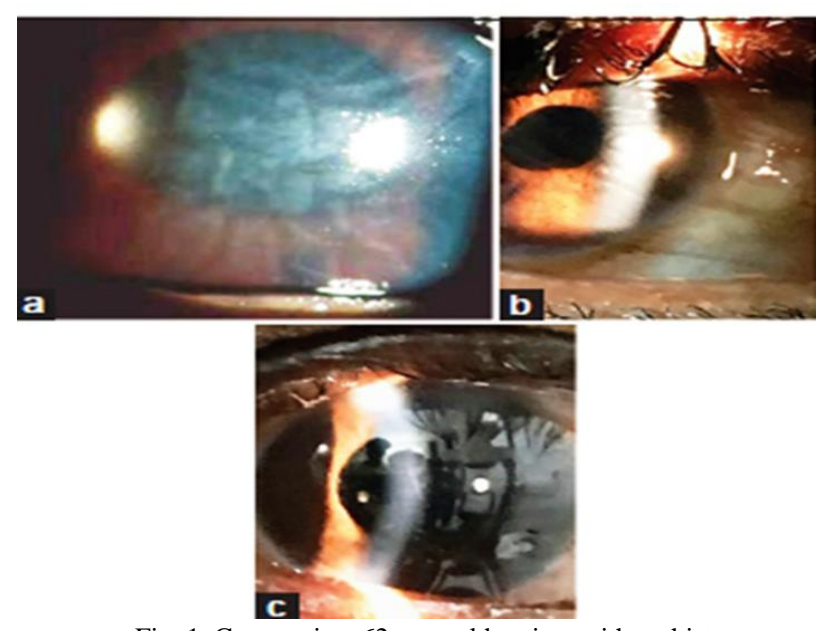

Fig. 1. Cataract in a 62-year-old patient with no history: a) fifth postoperative day: corneal edema, folds of Descemet's membrane and pigments on the endothelium;

b) fifteenth postoperative day: Decreased edema;

c) thirtieth postoperative day: Corneal lightening.

\section{DISCUSSION}

Since 1980, several cases of inflammation of the anterior segment have been reported after cataract surgery or associated with vitrectomy, this was explained by the introduction of toxic substances into the eye, and thus called sterile endophthalmitis defined in 1992 by Monson as Toxic Anterior Segment Syndrom, the inflammation sets in $12 \mathrm{~h}$ to $48 \mathrm{~h}$ postoperatively and almost all of our patients present with Visual Blur, Eye Pain, Corneal Edema and almost half have Thyndall, Hypopion, Retro-corneal Precipitates, Pupillary Cyclitic Membrane compared to others studies [1], in fact no paraclinical examination leads to the diagnosis of TASS and the only paraclinical examination leading to the elimination of the diagnosis of TASS is the positivity of the microbiological samples which indicates the presence of an infection.

Conversely, the negativity of the samples (approximately $30 \%$ of cases in the published series) [2] in no way eliminates the diagnosis of infectious endophthalmitis.

The exact cause of TASS remains unknown even after a thorough investigation [3] despite this - the inappropriate cleaning of surgical instruments is the most common cause of TASS according to the American Society for Cataract Surgery and Refractive Surgery (ASCRS) [4] as well as the intra-camerular injection of: Phenylephrine, cefuroxime and lidocaine May be a cause of TASS [5] also that Contamination with Balanced Salt Solution (BSS) may be a risk factor for TASS [6], Trypan blue used for the staining of the anterior crystalloid is incriminated to be the cause of TASS [7], [8] Contamination or denaturation of ViscoElastic Products may be involved [9] as well as metals heavy, also used in the composition of implants such as aluminum have been proposed as a possible cause of massive epidemic of subacute TASS in Japan [10].

Factors related to patients such as diabetes, high blood pressure, hyperlipidemia, chronic ischemic heart disease and chronic renal failure [11]. Recurrent uveitis as well as postoperative septic endophthalmitis and inflammation secondary to complicated surgery or persistence of intraocular lens residue are different from TASS.

The treatment consists of the use of steroid eye drops, the subconjunctival injection of dexamethasone and oral prednisolone as well as the washing of the anterior chamber, explanation, Anterior vitrectomy and Keratoplasty [7]-[10] The awareness and continuous training of surgical staff on TASS prevention protocols has shown good effectiveness.

\section{CONCLUSION}

In front of a painful red eye postoperatively after cataract surgery, is it always an endophthalmitis or a TAS syndrome, hence the place of antibiotic IVT in the slightest doubt as well as training surgical staff in the cleaning and sterilization of intraocular surgical instruments is a key element in the prevention of TAS Syndrom.

\section{REFERENCES}

[1] Anusha Koshal Ram, Bala Subramanian Toxic Anteriorsegment Syndrome Presenting as Endothelitis Following Uneventful Phaco Emulsification TNOA Journal of Ophthalmic Science and Research ! Volume 56 ; Issue 2! April-June 2018.

[2] Chiquet $\mathrm{C}$ and al. Eubacterial PCR for bacterial detection and identification in 100 acute postcataract surgery endophthalmitis Invest Ophthalmol Vis Sci 2008; 49.

[3] Moyle W, Yee RD, Burns JK, Biggins T. Two consecutive clusters of toxic anterior segment syndrome. Optom Vis Sci. 2013;90(1):e11-23.

[4] Mamalis N. Toxic anterior segment syndrome: role of enzymatic detergents used in the cleaning of intraocular surgical instruments. J Cataract Refract Surg. 2016;42(9):1249-50.

[5] Braga-Mele. Intracameral antibiotics: safety, efficacy, and preparation. J Cataract Refract Surg. 2014;40(12):2134-42.

[6] Andonegui J, Outbreak of toxic anterior segment syndrome after vitreous surgery. Arch Soc Esp Oftalmol. 2009;84(8):403-5.

[7] Matsou A, et al Generic trypan blue as possible cause of a cluster of toxic anterior segment syndrome cases after uneventful cataract surgery. J Cataract Refract Surg. 2017;43(6):848-52.

[8] Buzard K, et al. Two cases of toxic anterior segment syndrome from generic trypan blue. J Cataract Refract Surg. 2010;36(12):2195-9.

[9] Althomali TA. Viscoelastic substance in prefilled syringe as an etiology of toxic anterior segment syndrome. Cutan Ocul Toxicol. 2016;35(3):237-41.

[10] Oshika T, Eguchi S, Goto H, Ohashi Y. Outbreak of subacute-onset toxic anterior segment syndrome associated with single-piece acrylic intraocular lenses. Ophthalmology. 2017; 124:519-23.

[11] Yazgan S, et al: The role of patient's systemic characteristics and plateletcrit in developing toxic anterior segment syndrome after uneventful phaco surgery: a case-control study. Int Ophthalmol. 2018;38(1):43-52. 